Recherches amérindiennes au Québec

\title{
Coléoptères, poux et puces subfossiles provenant d'habitats de chasseurs-cueilleurs
}

L'apport des recherches archéoentomologiques dans le Nord circumpolaire

Subfossil beetles, lice and fleas from palaeoeskimo and neoeskimos sites The contribution of archaeoentomological research in the Circumpolar North

\section{Coleópteros, piojos y pulgas subfósiles provenientes de los hábitats de} cazadores-recolectores

\author{
El aporte de la investigación arqueo-entomológica en el norte \\ circumpolar
}

Véronique Forbes, Frédéric Dussault, Olivier Lalonde et Allison Bain

Volume 47, numéro 2-3, 2017

Les petites bêtes dans l'histoire et les cosmologies amérindiennes

URI : https://id.erudit.org/iderudit/1048592ar

DOI : https://doi.org/10.7202/1048592ar

Aller au sommaire du numéro

Éditeur(s)

Recherches amérindiennes au Québec

ISSN

0318-4137 (imprimé)

1923-5151 (numérique)

Découvrir la revue

Citer cet article

Forbes, V., Dussault, F., Lalonde, O. \& Bain, A. (2017). Coléoptères, poux et puces subfossiles provenant d'habitats de chasseurs-cueilleurs : l'apport des recherches archéoentomologiques dans le Nord circumpolaire. Recherches amérindiennes au Québec, 47(2-3), 11-21. https://doi.org/10.7202/1048592ar
Résumé de l'article

L'archéoentomologie, l'analyse des restes d'insectes préservés en contexte archéologique, est une approche relativement méconnue en Amérique du Nord. Bien qu'une première étude d'insectes subfossiles provenant d'un site paléoesquimau ait été conduite à la fin des années 1960 au Groenland, il s'est écoulé près de trois décennies avant que de telles études se réalisent dans l'Arctique nord-américain. Depuis, une vingtaine de sites de cultures paléoesquimaudes (Saqqaq, Dorset) et néoesquimaudes (thuléennes, inuites, yup'iks) ont fait l'objet d'analyses archéoentomologiques, produisant des milliers de spécimens de coléoptères, de poux et de puces. Ces données permettent d'examiner les pratiques de subsistance et d'hygiène des anciens peuples autochtones de l'Alaska, du Nord canadien et du Groenland, ainsi que leurs effets sur la faune et la flore locale. Cet article présente un bilan de l'apport des analyses archéoentomologiques à l'étude des modes de vie des chasseurs-cueilleurs du Nord circumpolaire.
Tous droits réservés @ Recherches amérindiennes au Québec, 2018

Ce document est protégé par la loi sur le droit d'auteur. L'utilisation des services d'Érudit (y compris la reproduction) est assujettie à sa politique d'utilisation que vous pouvez consulter en ligne.

https://apropos.erudit.org/fr/usagers/politique-dutilisation/ 


\section{Véronique Forbes*, Frédéric Dussault ${ }^{\star *}$, Olivier \\ Lalonde $^{* * *}$ et Allison Bain ${ }^{* * *}$}

* University of Aberdeen, School of Geosciences, Department of Archaeology, Aberdeen et UMR 5199 PACEA, Université de Bordeaux, France

** Memorial University of Newfoundland, Department of Archaeology, St. John's, NL

$* \star *$ Université Laval et CELAT, Québec

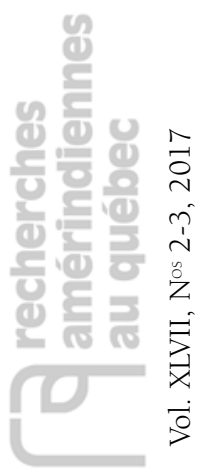

$\int_{\text {RÂCE AUX DÉCOUVERTES qui ont été faites }}$ J depuis le tout début de la recherche archéologique dans l'Arctique jusqu'à l'analyse récente d'ADN ancien de restes humains, nous savons que les ancêtres des Inuits et des Yupiks qui habitent aujourd'hui les régions nordiques de l'Amérique du Nord sont venus de Sibérie (Grønnow 2009; Hoffecker 2005; Raghavan et al. 2014). Nous les connaissons comme étant les Thuléens, descendants de populations originaires des régions autour du détroit de Béring, qui vinrent occuper un vaste territoire s'étendant de l'Alaska jusqu'au Groenland il y a environ 800 ans. Les Thuléens sont précédés par une population distincte, ayant elle aussi fait le voyage de la Sibérie jusqu'aux côtes des océans Arctique et Atlantique Nord, mais près de 2000 ans plus tôt. Ces mouvements de population sont à l'origine des deux grandes traditions archéologiques présentes dans les régions circumpolaires nord-américaines (fig. 1) : les Paléoesquimaux (regroupant les cultures Denbigh, Independence I, dorsétiennes, Groswater et Saqqaq) et les Néoesquimaux $^{1}$ (les cultures Birnik, Punuk et thuléennes). Après leur expansion dans l'est du territoire Arctique, les Thuléens côtoient les Dorsétiens, l'expression la plus récente de la tradition paléoesquimaude, dans le Nord canadien et au Groenland. Peu de temps après, soit autour de l'an 1200 de notre ère, ces derniers s'éteignent (Friesen 2004;
Hoffecker 2005). Des rapports hostiles entre les deux peuples et des conditions climatiques devenues difficiles sont les deux principaux arguments expliquant cette disparition (Friesen 2004; Maschner et al. 2009; Park 1993; Renouf 1999, et autres).

Bien que les cultures paléo- et néoesquimaudes représentent des populations culturellement et génétiquement distinctes, elles ont en commun leur mode de subsistance profondément ancré dans le cycle saisonnier. Tous ces peuples vivent de la chasse, de la pêche et de la cueillette et se déplacent pour suivre les ressources terrestres, marines et aquatiques disponibles selon les saisons. Puisque la vaste région qu'ils occupent comprend une variété d'écosystèmes (de la toundra au désert polaire), ils développent diverses innovations technologiques leur permettant d'exploiter plus efficacement les ressources locales (Friesen et Mason 2016). Sur les sites de culture paléoesquimaude, on retrouve un outillage lithique et osseux ainsi que des ossements d'animaux qui démontrent que ces groupes s'adonnaient à la chasse aux grands mammifères tels le bouf musqué, le caribou, le morse et le phoque, en plus de pratiquer la pêche et la chasse aux oiseaux. En hiver, ils vivent dans des igloos ou des maisons semi-souterraines faites de tourbe (Hoffecker 2005; McGhee 2001; Renouf 2003). Les modes de vie 


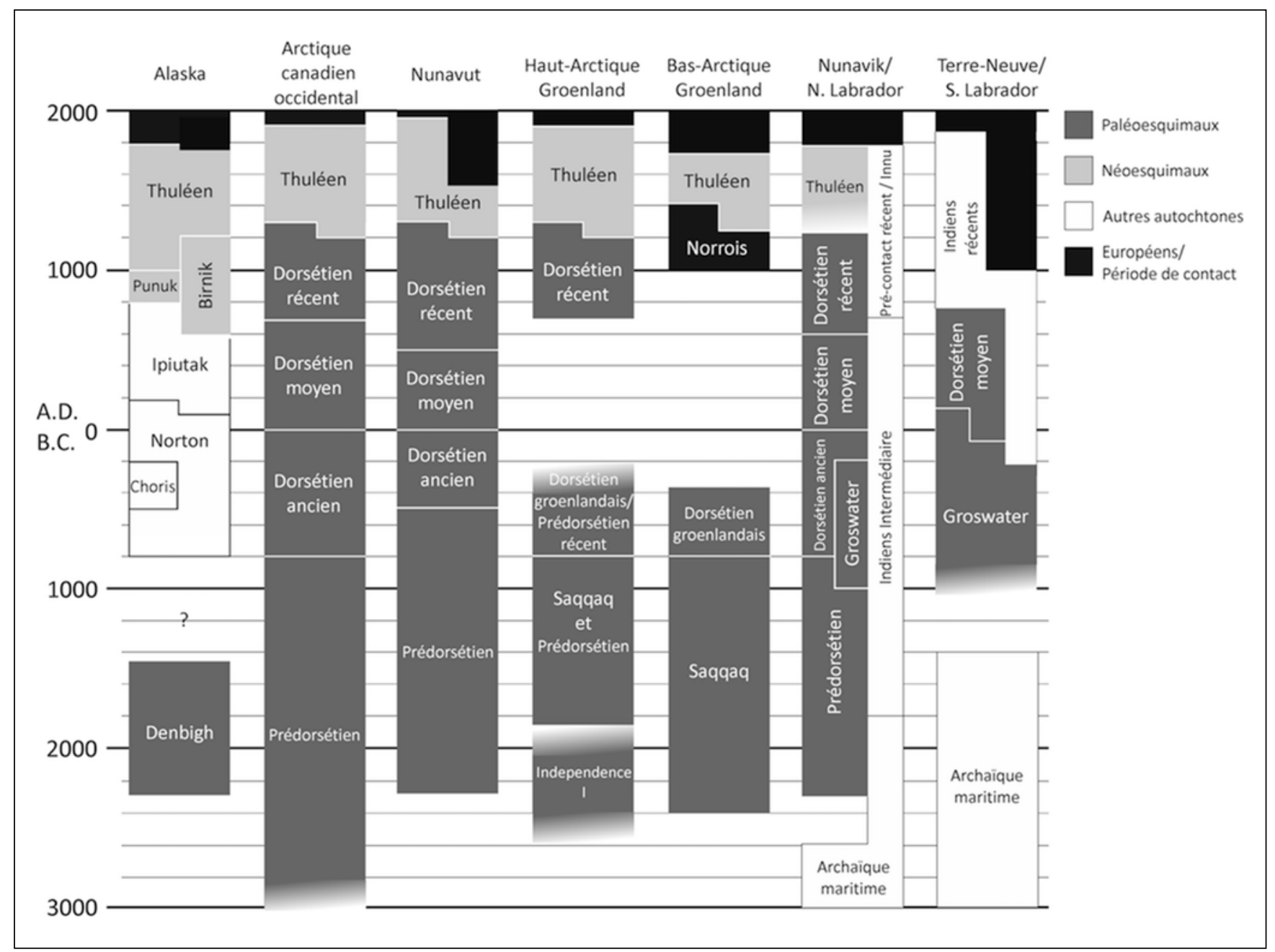

\section{Figure 1}

Chronologie des peuples des régions circumpolaires nord-américaines

(Basé sur les chronologies présentées dans Raghavan et al. 2014 et par l'Institut culturel Avataq 2015)

néoesquimaux sont, quant à eux, caractérisés par une plus grande emphase sur les ressources marines et le développement d'outils spécialisés incluant l'umiak (large embarcation à pagaie), la tête de harpon basculante à logette (toggle-head harpoon), le filet de pêche avec poids et le flotteur en peau (drag float) servant à la chasse aux mammifères marins. Ils introduisent aussi les traîneaux à chiens et une nouvelle forme de maison semi-souterraine comprenant un tunnel d'entrée en contrebas qui permet de mieux réguler la température intérieure (Kleivan 1978; Park 1999; Schledermann 1976).

Dans les régions les plus septentrionales de l'Arctique, le faible niveau d'enfouissement de certains sites archéologiques peut faire que seuls les matériaux durables comme la pierre, l'os et l'andouiller sont préservés. De fait, une large part de notre connaissance des modes de vie des chasseurscueilleurs de l'Arctique provient de l'étude des technologies et des ossements d'animaux. Ces données nous permettent de mieux comprendre le rôle prédominant de la chasse et de la pêche dans la subsistance, mais elles peuvent difficilement nous renseigner sur certains autres aspects des modes de vie. Par exemple, quelle était l'ampleur de la place occupée par les ressources végétales dans l'alimentation de ces communautés et de quelle(s) manière(s) se les procuraient-elles? Comment se déroulait la vie quotidienne dans leurs habitats - notamment, comment s'organisaient la préparation de la nourriture et des matériaux bruts, la fabrication et l'entretien des habitations et des outils, la disposition des déchets et les pratiques d'hygiène dans l'espace domestique? Quels étaient les effets de ces pratiques sur l'environnement local? L'ethnographie et la tradition orale nous permettent d'esquisser des réponses possibles à ces questions (par ex. Alix et Brewster 2004; FienupRiordan 2007; Rasmussen 1929). Néanmoins, on ne peut assumer que les descriptions contenues dans ces sources s'appliquent au passé plus distant ou à un site particulier, sans les avoir vérifiées au préalable avec les données archéologiques appropriées. Or, sur de nombreux sites localisés dans la zone circumpolaire, la présence de pergélisol, de tourbière ou d'autres types de matière organique humide (par exemple les restes de boucherie et autres débris formant les dépotoirs archéologiques) crée les conditions 
idéales pour la préservation de matériaux périssables tels le bois, les graines, les fruits et les insectes (par ex. Alix 2004; Forbes et al. 2015; Zutter 2009, 2012). Tous ces artefacts et «écofacts » représentent d'excellentes occasions d'examiner les modes de vie de ceux qui ont vécu autrefois en ces lieux.

Les recherches conduites par quelques archéobotanistes ont permis de mieux comprendre la saisonnalité de l'occupation de certains sites ainsi que l'utilisation des ressources végétales comme combustible, nourriture et matière première (Alix 2004; Crawford 2012; Lepofsky et al. 2001; Zutter 2009, 2012). D’autres ont interprété l'empreinte écologique de ces populations sur l'environnement local à partir de divers types de données paléoenvironnementales. Par exemple, les résultats de recherches conduites sur divers sites situés au sud de la limite forestière dans le Labrador septentrional ont servi à documenter l'exploitation intensive du bois par les Inuits du XvII au XIX ${ }^{e}$ siècle ainsi que l'impact environnemental d'une telle exploitation (LemusLauzon et al. 2012, 2016; Roy et al. 2012; Roy et al. 2015). Lanalyse de pollens et chironomes subfossiles provenant de Bass Pond, sur la côte nord-ouest de Terre-Neuve, a aussi révélé des changements écologiques interprétés comme résultant de l'occupation dorsétienne de la région (Renouf 2011; Renouf et Bell 2009). Lorsqu'elles sont intégrées dans un contexte de recherche multidisciplinaire, ces reconstitutions peuvent grandement aider à mettre en lumière la complexité des dynamiques homme-environnement à la base des modes de vie des peuples nordiques (par ex. Kaplan et Woollett 2000; Woollett 2007). Cependant, tant que ces efforts demeureront peu nombreux et géographiquement fragmentés, il sera difficile de comprendre comment ces populations ont véritablement vécu et su se montrer résilientes dans cette région apparemment inhospitalière aux autres peuples nord-américains.

Larchéoentomologie, l'analyse des exosquelettes d'insectes extraits de sédiments archéologiques, est une approche méconnue qui se révèle particulièrement efficiente pour comprendre certains aspects des modes de vie paléo- et néoesquimaux. Cet article présente une introduction à l'archéoentomologie et dresse un premier bilan des résultats des analyses conduites jusqu'à présent dans les régions circumpolaires du continent américain.

\section{L'ARCHÉOENTOMOLOGIE : MÉtHODE ET PRATIQUE}

L'« entomologie du Quaternaire » (Quaternary entomology) est une sous-discipline qui comprend les approches dites «paléoentomologique » et " archéoentomologique ». La distinction entre ces deux entités relève davantage d'une différence dans leur application que d'une véritable disparité dans leurs bases théoriques et méthodologiques. En effet, alors que les études dites paléoentomologiques visent à reconstituer les changements climatiques et environnementaux résultant ou non de causes naturelles (par ex. Elias et Matthews 2002; Lavoie et Arseneault 2001; Ponel et al. 2005), les études archéoentomologiques se concentrent sur les environnements anthropisés et cherchent à examiner les pratiques culturelles et les interactions humainenvironnement (par ex. Dussault, Bell et Grimes 2016; Forbes et al. 2014; Huchet 1996; Ponel et al. 2013; Smith 2012). La paléoentomologie et l'archéoentomologie se basent donc sur les mêmes principes et méthodologie et fondent leurs reconstitutions sur les exigences biologiques et écologiques des espèces d'insectes extraites d'échantillons de sédiments ou de tourbe (Elias 2010). Il s'agit, la plupart du temps, d'espèces qui existent encore aujourd'hui, puisqu'il a été démontré que les insectes n'ont que peu ou pas évolué depuis les derniers 2,5 millions d'années (Coope 1978)

La méthodologie à suivre pour recueillir des échantillons et en extraire les restes entomologiques est relativement simple. Il s'agit de collecter quelques litres de sédiments avec des outils propres et de les placer dans un contenant fermé hermétiquement. Ces sédiments doivent idéalement être entreposés dans un milieu sombre et frais jusqu'à leur traitement en laboratoire. Pour extraire les restes d'insectes, les échantillons sont soumis à la flottation au kérosène (paraffin flotation), un procédé qui fut développé au Royaume-Uni à la fin des années 60 (Coope et Osborne 1967). Il consiste d'abord à désagréger les échantillons dans l'eau et à les filtrer à 250 ou $300 \mu$ avant de les drainer et de les mélanger avec leur volume égal de kérosène. De l'eau froide est ensuite ajoutée à la mixture et le tout est laissé au repos de 10 à 30 minutes, le temps de permettre aux parties d'insectes, enduites de kérosène, de remonter à la surface. La fraction flottante est alors décantée dans un tamis et lavée au détergent. À l'aide d'un microscope à faible grossissement (10X), il est possible d'examiner cette fraction et d'en extraire les restes d'insectes.

La prochaine étape de l'analyse est l'identification des restes à l'espèce, ou, le cas échéant, au plus haut niveau taxonomique possible. Il est plus ardu d'identifier des insectes subfossiles (fragments d'insectes préservés dans les sédiments naturels et culturels) que des insectes modernes, puisque les parties molles sont habituellement décomposées, entraînant la désarticulation de l'exosquelette. Pour cette raison, les identifications sont normalement effectuées au moyen de comparaisons morphologiques directes avec des spécimens provenant d'une bonne collection de référence (Elias 2010), idéalement composée de spécimens récents ainsi que subfossiles identifiés à l'espèce.

\section{L'ARCHÉOENTOMOLOGIE DES SITES PALÉO- ET NÉOESQUIMAUX}

La toute première étude archéoentomologique sur le territoire circumpolaire nord-américain a été conduite au Groenland à la fin des années 1960 (Haarløv 1967). Il fallut attendre un quart de siècle avant que d'autres travaux similaires aient lieu dans cette région (Bresciani et al. 1983; 1989; Hansen et al. 1991). À partir des années 1990, l'archéoentomologie commence à se développer en Amérique du Nord, coincidant avec les fouilles d'occupations dorsétiennes et inuites sur l'île de Baffin (voir Bain 1994, 1997, 


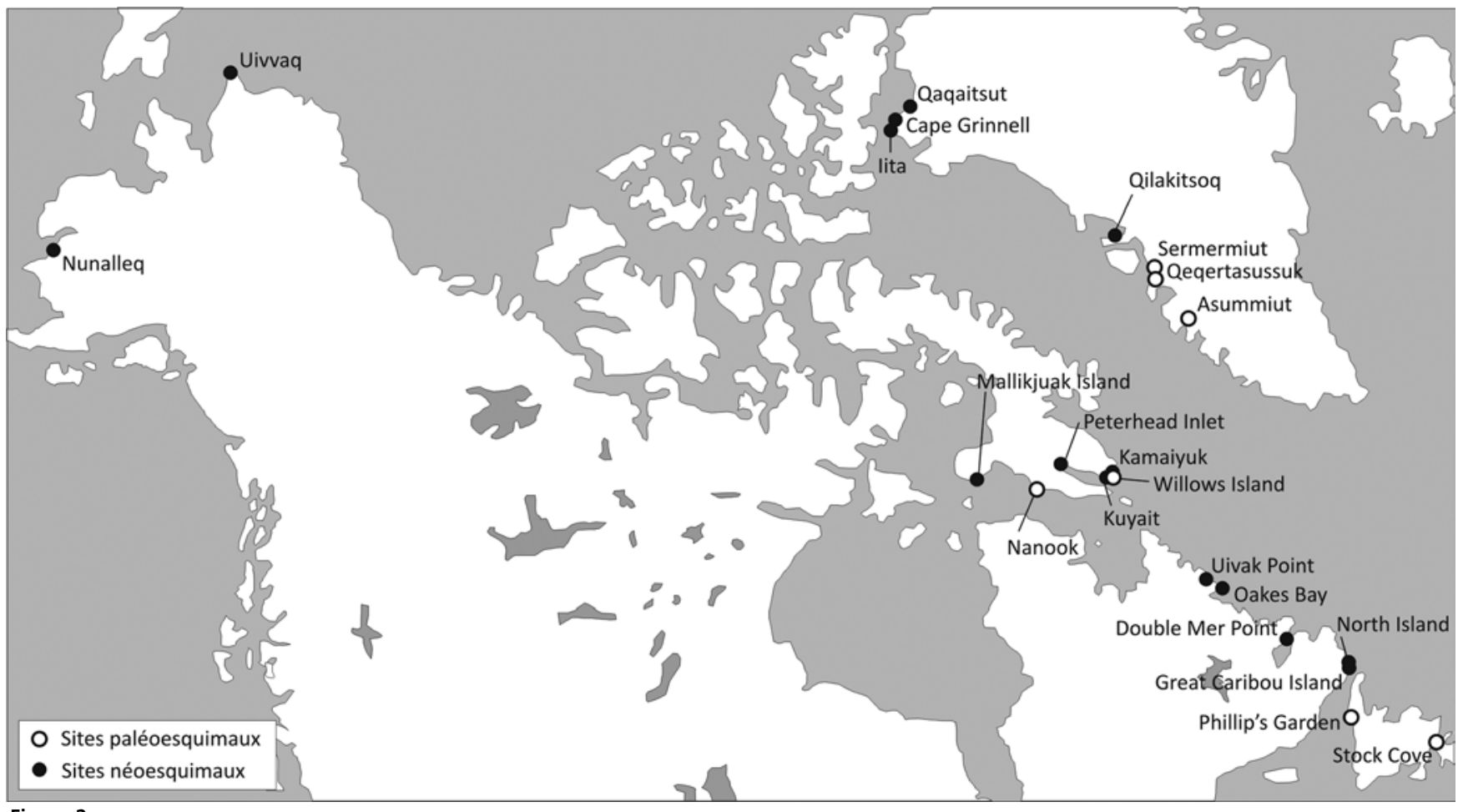

Figure 2

Location des sites archéologiques paléo- et néoesquimaux ayant fait (ou faisant présentement) l'objet d'analyses archéoentomologiques

1998; Smith 1992, 1994). Par la suite, quelques assemblages provenant de sites situés au Labrador, à Terre-Neuve et en Alaska sont également examinés (Bain 2000, 2001b; Dussault et Bain 2010; Dussault, Bell et Grimes 2016; Forbes et al. 2015; Hoffecker et al. 2012).

La localisation de l'ensemble des sites paléo- et néoesquimaux ayant fait l'objet d'analyses archéoentomologiques est présentée dans la figure 2. Dans la plupart des cas, il s'agit de sites d'occupation: soit des maisons d'hiver, soit des dépotoirs archéologiques contenant des restes de boucherie et autres détritus de la vie quotidienne. Bien que plusieurs de ces projets soient toujours en cours, les données obtenues jusqu'à présent permettent d'apprécier l'apport et le potentiel de l'archéoentomologie en archéologie de l'Arctique. Les résultats obtenus jusqu'à présent permettent l'examen de deux thématiques principales: (i) les pratiques domestiques et aires d'activité et (ii) la relation entre les chasseurs-cueilleurs et leur environnement.

\section{Pratiques domestiques et aires \\ D'ACTIVITÉ : LES ECTOPARASITES}

Le terme « ectoparasite » désigne un groupe écologique, essentiellement hématophage ou détritiphage (se nourrissant de sang ou de détritus), composé d'insectes, d'arachnides et de crustacés vivant à la surface de la peau, dans la fourrure ou sur les plumes d'oiseaux (Hopla et al. 1994; Marshall 1981). Lorsque les conditions de préservation sur les sites archéologiques sont optimales, par exemple lorsqu'il y a présence de pergélisol ou que les dépôts échantillonnés sont demeurés constamment humides depuis leur enfouissement, le pou de l'homme, Pediculus humanus L. (fig. 3a), est presque toujours présent (Forbes et al. 2013). Cet insecte, un ectoparasite spécialisé se nourrissant exclusivement de sang humain (Durden 2002), a été trouvé dans des niveaux d'occupation appartenant à des sites inughuits et thuléens au Groenland (à Cape Grinnell, Qaqaitsut et Iita, Dussault et al. 2014), au Labrador (à Uivak Point, A. Bain, comm. pers., 2016) et en Alaska (à Nunalleq, Forbes et al. 2015). Lune des découvertes les plus remarquables provient de momies groenlandaises de Qilakitsoq, datées du $\mathrm{XV}^{\mathrm{e}}$ siècle. Plusieurs spécimens du pou de l'homme ont été retrouvés non seulement dans les cheveux, mais aussi à l'intérieur de l'estomac de ces momies, suggérant que les insectes étaient avalés lors de l'épouillage (Bresciani et al. 1983, 1989; Hansen et al. 1991). Plusieurs sources ethnographiques portant sur les traditions inuites mentionnent que les poux pouvaient être écrasés entre les dents ou avalés (voir Laugrand et Oosten 2010). Les poux de Qilakitsoq attestent donc l'antiquité de cette pratique.

Les poux ne sont associés qu'avec une ou quelques espèces d'hôtes étroitement apparentées et ils passent toute leur vie attachés à la peau, aux cheveux, à la fourrure ou aux vêtements de cet hôte, ne le quittant qu'à la mort ou pour trouver un nouvel hôte (Marshall 1981). Pour cette raison, on ne va pas nécessairement retrouver une grande quantité de poux sur les sols de bâtiments ayant servi à loger les hôtes de ces ectoparasites. Le fait que les analyses archéoentomologiques conduites dans le nord de l'Europe ont produit peu d'ectoparasites de bétail dans du fumier provenant d'étables et peu de poux de l'homme dans les dépôts d'occupation 
provenant de maisons, illustre bien ce fait (Nielsen et al. 2000; Forbes et Milek 2014; Kenward 2009). Ce n'est pas la simple présence de l'hôte mais plutôt les pratiques impliquant l'enlèvement intentionnel des ectoparasites qui sont susceptibles de créer d'importantes concentrations dans les dépôts archéologiques. C'est sur la base de ce principe que des aires d'activités pour l'épouillage ont été identifiées sur quelques sites norrois (Buckland et al. 1992, 1998). Les fortes concentrations d'ectoparasites de l'homme identifiées dans les tunnels d'entrée des maisons inughuites étudiées à Cape Grinnell et à Qaqaitsut au Groenland ont également été interprétées comme attestant des pratiques d'épouillage. Le fait que les cadavres de poux aient été jetés en dehors des plateformes de couchages et des aires de vie semble dénoter un certain souci de garder l'espace domestique hygiénique (Dussault et al. 2014), contrastant avec les perceptions véhiculées dans certains récits d'explorateurs (par ex. Henson et al. 1912; Peary 1898; Peary et Peary, 1893). Les habitants d'Uivak Point au Labrador semblent aussi s'être adonnés à l'épouillage, comme le démontrent des analyses récentes qui révèlent une concentration de poux sans tête autour d'un pied de lampe se trouvant dans l'aire d'activité principale d'une maison (Bain, comm. pers., 2016).

Outre le pou de l'homme, un autre «indésirable», le morpion Phthirus pubis (L.) [fig. 3b], semble avoir cohabité avec les ancêtres des Inuits. À ce jour, seul trois spécimens ont été identifiés dans des maisons inughuites/thuléennes à Cape Grinnell, au Groenland (Dussault et al. 2014). De récentes analyses sur le site précontact yup'ik de Nunalleq en Alaska ont aussi livré plusieurs spécimens d'un autre ectoparasite, la puce de l'homme, Pulex irritans L. (fig. 3c). Il s'agit des seuls spécimens de cette espèce jamais enregistrés en Alaska. Généralement, les puces sont beaucoup plus mobiles et moins spécifiques à leur hôte que les poux. Par exemple, la puce de l'homme est surtout associée à l'être humain, mais on lui connaît aussi d'autres hôtes, incluant le cochon, le chien de prairie, le coyote et même la chouette des terriers (Holland 1985). Pour cette raison, les puces subfossiles sont généralement moins utiles pour reconstituer les activités domestiques et économiques ayant eu lieu sur un site. Les subfossiles de Nunalleq fournissent tout de même un nouvel élément venant appuyer le modèle biogéographique proposé pour la dispersion de la puce de l'homme. En effet, la phylogénie de P. irritans ainsi que les données archéoentomologiques accumulées jusqu'à présent suggèrent que cette espèce serait originaire d'Amérique du Sud et qu'elle aurait atteint l'Europe il y a plus de 5000 ans, vraisemblablement après être passée par le détroit de Béring (Buckland et Sadler 1989; Yvinec et al. 2000).

Les ectoparasites d'un animal domestique ayant joué un rôle primordial dans le mode de vie des chasseurs-cueilleurs de l'Arctique sont aussi présents sur certains sites. En effet, à Cape Grinnell et à Qaqaitsut au Groenland, quelques spécimens du pou suceur du chien Linognathus setosus von Olfers (fig. 3d) ont été découverts (Dussault et al. 2014). À Nunalleq en Alaska, c'est le pou broyeur du chien Trichodectes canis (De Geer) [fig. 3e] que l'on trouve en grand nombre (Forbes et al. 2015). Il est possible que la présence de ces poux dans les niveaux d'occupation des maisons soit indicative des lieux où les chiens étaient admis à l'intérieur des habitations (cf. Dussault et al. 2014). À Nunalleq, d'importantes variations dans les concentrations de poux broyeurs du chien dans les différentes parties de la structure de tourbe ont pu être observées. De même, des marques de boucherie indicatives de l'enlèvement des peaux ont été identifiées sur certains os de canidés (McManus 2015). Selon les sources ethnographiques de la région (par ex. Fienup-Riordan 2007), les méthodes traditionnelles pour le traitement des peaux impliquaient leur vieillissement et/ou leur raclement pour en enlever la fourrure. Comme de telles pratiques délogeaient sans doute les ectoparasites en plus 
d'enlever les poils, il est possible que la distribution spatiale des poux de chien à Nunalleq soit le résultat du traitement des peaux de canidés.

Un pou Nirmus sp. (fig. 3f) et quelques puces d'oiseaux Ceratophyllus vagabundus (Boheman) complètent la liste des ectoparasites trouvés en contextes paléo- et néoesquimaux. Ces restes, issus de sites groenlandais, ont été interprétés comme provenant d'oiseaux chassés pour la subsistance (Böcher et Fredskild 1993; Dussault et al. 2014). Comme les poux d'oiseaux restent logés entre les plumes de leurs hôtes tout au long de leur cycle de vie (Marshall 1981), ces insectes se sont vraisemblablement retrouvés sur les sols des habitations inughuites après la préparation de carcasses (Dussault et al. 2014). Puisque les puces du genre Ceratophyllus passent la plus grande partie de leur cycle de vie non pas directement sur leurs hôtes mais plutôt dans leurs nids (Marshall 1981; Rothschild et Clay 1957), il est aussi possible que la récolte de ressources provenant de tels milieux (par ex. duvet) explique la présence de puces d'oiseaux dans les sites archéologiques (par ex. Forbes 2015).

\section{LA RELATION ENTRE LES CHASSEURS-CUEILLEURS ET L'ENVIRONNEMENT NORDIQUE : LES COLÉOPTĖRES}

Les coléoptères sont les insectes les plus étudiés par les archéoentomologues non seulement parce que leurs exosquelettes sont particulièrement durables, mais aussi parce qu'ils occupent tous les écosystèmes terrestres et aquatiques et qu'ils ont des exigences environnementales caractéristiques (Elias 2010). Chaque espèce occupe une niche écologique particulière et nécessite des conditions de température spécifiques pour pouvoir compléter son cycle de vie (passer du stade de la larve jusqu'à celui de l'adulte reproductif). Par exemple, plusieurs coléoptères phytophages se nourrissent exclusivement des tissus végétaux provenant d'une ou de quelques plantes hôtes, alors que les coléoptères coprophages requièrent la présence d'excréments pour se nourrir. Le compost, les charognes, les nids, la litière forestière, les denrées entreposées, les cours d'eau et les débris sur la zone littorale sont quelques exemples d'autres niches écologiques colonisées par différentes espèces de coléoptères (Crowson 1981). Outre ces exigences, la capacité ou non pour une espèce de vivre et de se reproduire dans un lieu particulier est déterminée par des contraintes climatiques (Coope et al. 1977). En effet, chaque espèce peut être classée selon ses préférences pour des températures estivales chaudes, tempérés ou fraîches, et pour des climats océaniques ou continentaux (Atkinson et al. 1986). Ainsi, les coléoptères subfossiles préservés en association avec des vestiges archéologiques peuvent permettre de reconstituer les conditions environnementales et climatiques locales contemporaines à leur occupation. Ce fut d'ailleurs l'objectif de plusieurs études conduites dans les régions septentrionales (Haarløv 1967; Böcher et Fredskild 1993; Elias 2003). Par exemple, l'étude effectuée par Böcher et Fredskild (1993) sur le site de Qeqertasussuk au Groenland a permis d'identifier plusieurs espèces de coléoptères se trouvant bien au-delà de leur limite géographique actuelle, indiquant qu'à l'époque de l'occupation Saqqaq du site (il y a de cela trois millénaires), les conditions climatiques étaient plus chaudes qu'aujourd'hui.

Sur la vingtaine de sites européens de la côte est de l'Amérique du Nord ayant fait l'objet d'analyses archéoentomologiques, on trouve une large proportion (généralement plus du tiers des taxons de coléoptères identifiés) d'espèces exotiques (non indigènes) introduites à la suite de la colonisation européenne et les échanges transatlantiques (Bain 1998, 2001a; Bain et King 2011; Bain et Prévost 2010). À cet égard, l'une des plus grandes différences entre les faunes de coléoptères subfossiles provenant de ces contextes et ceux provenant des sites paléo- et néoesquimaux est la rareté, voire l'absence totale sur la majorité des sites, d'espèces exotiques. Les seuls coléoptères originaires de la région paléarctique (la zone écologique comprenant l'Europe, l'Afrique du Nord ainsi qu'une partie de l'Asie et du Moyen-Orient) à avoir été identifiés sont Latridius minutus (L.) [fig. 4a] et Omalium rivulare (Paykull) [fig. 4b] (Dussault et Bain 2010 ; Lalonde 2015, 2016). L. minutus est un petit coléoptère mycétophage (se nourrissant de moisissures et champignons) associé à la matière végétale en décomposition et commun dans les étables et les entrepôts (Bousquet 1990; Forbes et al. 2016; Klimaszewski et al. 2010). En milieu tempéré, cet insecte est capable d'exploiter des habitats naturels tels que les souches et l'écorce d'arbres morts et les nids et terriers de mammifères, d'oiseaux et d'autres insectes (Hinton 1941; Majka et al. 2009). Le staphylin $O$. rivulare est aussi capable d'exploiter une variété d'habitats, mais en Amérique du Nord, on le retrouve surtout en association avec les charognes et la matière végétale en décomposition (Hatch 1957). Les restes subfossiles de L. minutus et de O. rivulare proviennent tous de sites du Labrador, plus spécifiquement de Great Caribou Island et Double Mer Point, tous deux postérieurs à la période de contact. Les autochtones du Nord-Est canadien ont commencé à entretenir des échanges avec les Européens depuis la venue des pêcheurs basques au XVI siècle (Pintal et al. 2015), avant l'arrivée des premiers missionnaires (Taylor 1974). L. minutus et O. rivulare ont donc probablement été introduits au Labrador par l'entremise de ces contacts. Bien qu'il semble que ces espèces ne soient pas parvenues à établir de populations permanentes au Labrador, ces données soulignent le rôle potentiel qu'ont pu jouer les échanges entre les autochtones et les Européens dans les transferts biologiques de longue-distance causés par la rencontre de l'Ancien et du Nouveau Monde.

Bien que les faunes coléoptères des sites paléo- et néoesquimaux soient principalement formées d'espèces indigènes faisant partie des communautés écologiques de la toundra, de la taïga et des plaines côtières, elles apparaissent différentes de celles trouvées dans les milieux naturels environnants. Ces assemblages sont presque toujours dominés par des coléoptères indicatifs de conditions particulièrement humides et riches en matière organique (voir tableau). Leur prédominance dans les dépôts archéologiques suggère 


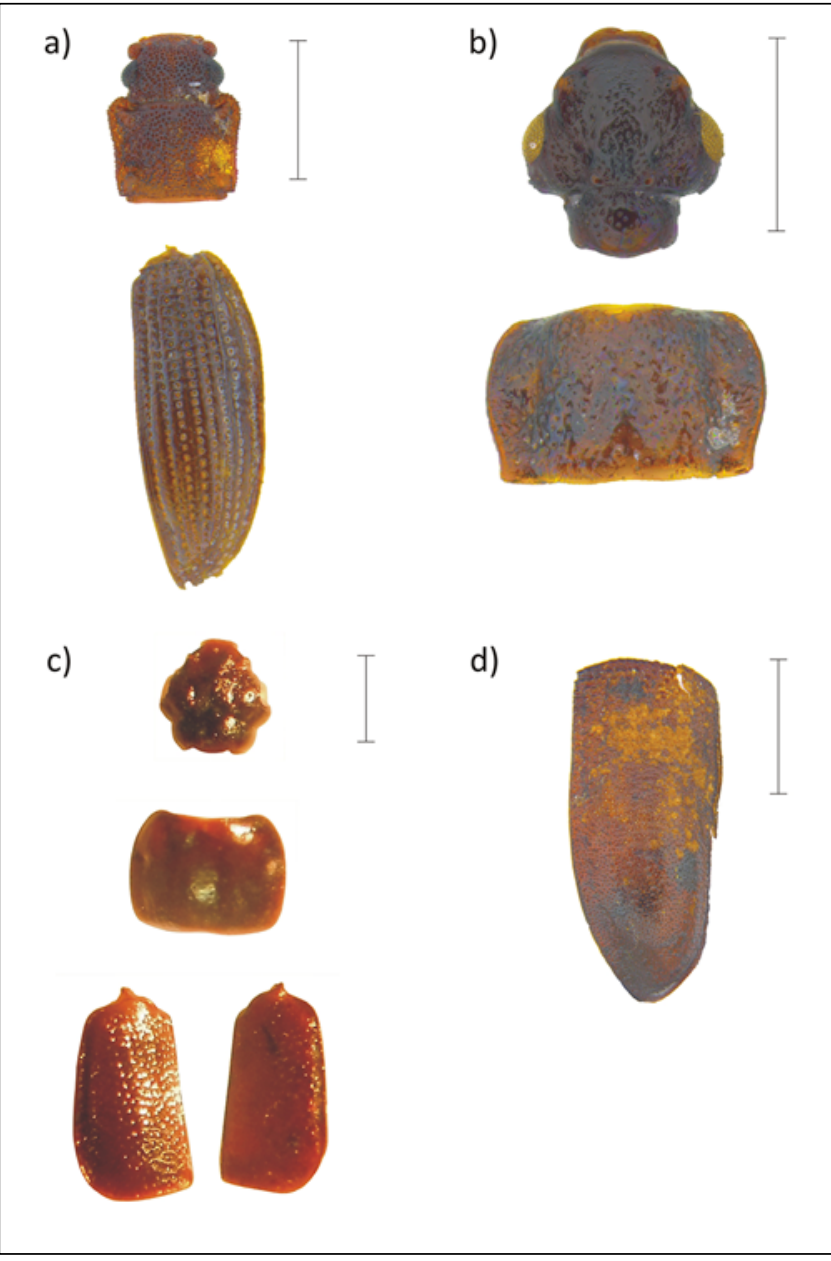

Figure 4

Quelques spécimens de coléoptères subfossiles

a) tête, pronotum et élytre de Latridius minutus de Double Mer Point,

Labrador; b) tête et pronotum d'Omalium rivulare de Double Mer

Point, Labrador; c) tête, pronotum et élytres d'Eucnecosum sp. de

Nunalleq, Alaska; d) élytre de Polygraphus rufipennis de Phillip's

Garden, Terre-Neuve

(Échelle : 0,5 mm)

que ces sites archéologiques et les activités qui y étaient pratiquées constituaient des environnements anthropiques où la matière organique humide abondait. Lutilisation de tourbe comme matériau de construction et le travail du bois et des carcasses animales favorisaient sans doute la création de micro-habitats idéals pour certaines espèces indigènes normalement confinées aux débris sur la zone littorale ou à d'autres milieux particulièrement humides et riches en nutriments. On remarque aussi que plusieurs des taxons les plus abondants sont capables d'exploiter les niches écologiques se trouvant dans les nids et les terriers de mammifères et d'oiseaux. C'est le cas notamment des staphylins du genre Eucnecosum (fig. 4c), qui sont extrêmement nombreux dans les contextes archéologiques de Nunalleq, en Alaska et à Uivak Point, au Labrador. Lintérieur des maisons de tourbe, humide et riche en matière organique, évoque les micro-habitats des nids et terriers que ces insectes occupent originellement (cf. Kenward et Allison 1994).
Une large proportion des espèces de coléoptères vivant aujourd'hui en association étroite avec l'être humain semblent avoir vécu dans les réserves de nourriture des nids de rongeurs et d'oiseaux avant de coloniser nos maisons et entrepôts à grain (Kenward et Allison 1994; King 2014). Parmi celles-ci, on retrouve des espèces ravageuses, dont le charançon du blé, Sitophilus granarius (L.), qui semble dépendant des réserves de grains entreposés. Les plus anciens spécimens de $S$. granarius et de ses congénères S. oryzae (L.) et S. zeamais Motschulsky proviennent de contextes du Néolitique (PPNC) au Moyen-Orient (Helbaek 1970) et de la période Jōmon au Japon (Obata et al. 2011). Pour cette raison, on assume que l'origine de la synanthropie chez les insectes coinnciderait avec les débuts de l'agriculture et des premiers stockages des céréales (Buckland 1981; Plarre 2010). Sachant que plusieurs des taxons présents sur les sites paléo- et néoesquimaux sont capables d'exploiter les nids et terriers de rongeurs, on serait à même de penser que la transition des nids et terriers aux habitations humaines se soit faite beaucoup plus tôt. Seule l'analyse de sites antérieurs au Néolithique viendrait confirmer ou infirmer cette hypothèse. Il est intéressant toutefois de mentionner que chez les Yupiks de l'Alaska, il était coutume de «piller» les nids de rongeurs pour en recueillir certaines plantes (Ager et Ager 1980; Anderson 1939). Si les ancêtres des Yupiks et des Inuits ont aussi exploité les réserves de nourriture de mammifères présents dans leur environnement, il est fort possible que cela ait favorisé l'introduction de coléoptères nidicoles à l'intérieur de leurs maisons.

Outre les coléoptères attirés par les niches générées dans les habitats des chasseurs-cueilleurs, on retrouve aussi ceux qui sont passivement transportés dans les blocs de tourbe, la mousse, le bois, les plantes et les ressources aquatiques utilisées. Il ne fait aucun doute que certain des dytiques, carabes et coléoptères phytophages identifiés dans les assemblages archéologiques ont été introduits dans les sites d'occupation de cette manière. Sur le site historique inuit de Uivak Point au Labrador, la présence combinée du coléoptère Polygraphus rufipennis (Kirby) [fig. 4d] et de brindilles et branches de conifères dans des échantillons provenant des maisons d'hiver suggère que les habitants exploitaient activement les ressources forestières locales pour aménager leur espace de vie (Bain 2000, 2001b; Zutter 2009, 2012). Le paysage côtier du Labrador où sont situés ces sites est aujourd'hui ouvert et dénué d'arbres, ce qui pourrait être une conséquence de l'exploitation des ressources végétales par les Inuits.

\section{Conclusion}

Les recherches archéoentomologiques conduites jusqu'à présent dans les régions arctiques et subarctiques démontrent l'utilité de l'étude des restes d'insectes subfossiles dans la reconstruction des modes de vie paléo- et néoesquimaux. Les ectoparasites tels les poux et les puces témoignent de pratiques hygiéniques (épouillage) et de 


\section{Liste et classement des taxons de coléoptères les plus abondants sur trois sites paléo- et néoesquimaux}

\begin{tabular}{|c|c|c|c|}
\hline SITES ARCHÉOLOGIQUES & $\overline{\text { CLASSEMENT }}$ & TAXON & HABITAT(s) \\
\hline \multirow{4}{*}{$\begin{array}{l}\text { Nunalleq, sud-ouest de l'Alaska, } \\
\text { xvile siècle (V. Forbes, analyses en cours, } \\
\text { données non publiées) }\end{array}$} & 1 & Aleocharinae indet. & $\begin{array}{l}\text { Feuilles mortes et autres débris végétaux, bois pourri, champignons, } \\
\text { charognes, excréments, nids et terriers (Arnett et Thomas 2001) }\end{array}$ \\
\hline & 2 & Pycnoglypta spp. & $\begin{array}{l}\text { Débris végétaux et à la base des plantes dans les marais et tourbières, } \\
\text { parfois dans les nids de rongeurs (Campbell 1983) }\end{array}$ \\
\hline & 3 & Eucnecosum spp. & $\begin{array}{l}\text { Débris végétaux et à la base des plantes dans les marais et tourbières, } \\
\text { parfois dans les nids de rongeurs ou d'oiseaux (Campbell 1984) }\end{array}$ \\
\hline & 4 & Acrotrichinae indet. & $\begin{array}{l}\text { Feuilles mortes et autres débris végétaux, nids et terriers de mammifères, } \\
\text { excréments, champignons (Arnett et Thomas 2001) }\end{array}$ \\
\hline \multirow[t]{3}{*}{$\begin{array}{l}\text { Uivak Point, Labrador, xVIII-xIxe siècles } \\
\text { (A. Bain, données non publiées) }\end{array}$} & 1 & Aleocharinae indet. & $\begin{array}{l}\text { Feuilles mortes et autres débris végétaux, bois pourri, champignons, } \\
\text { charognes, excréments, nids et terriers (Arnett et Thomas 2001) }\end{array}$ \\
\hline & 2 & $\begin{array}{l}\text { Eucnecosum brunnescens } \\
\text { (J. Sahlberg) }\end{array}$ & $\begin{array}{l}\text { Débris végétaux et à la base des plantes dans les marais et tourbières, } \\
\text { parfois dans les nids de rongeurs ou d'oiseaux (Campbell 1984) }\end{array}$ \\
\hline & 3 & $\begin{array}{l}\text { Helophorus arcticus } \\
\text { (W.J. Brown) }\end{array}$ & $\begin{array}{l}\text { Près des rivages sur les sols humides, dans la mousse et sous les pierres } \\
\text { (Smetana 1988) }\end{array}$ \\
\hline \multirow{5}{*}{$\begin{array}{l}\text { Qeqertasussuk, ouest du Groenland, } \\
\text { "e millénaire av. J.-C. (Böcher et Fredskild } \\
\text { 1993; Buckland et Buckland 2006) }\end{array}$} & 1 & $\begin{array}{l}\text { Micralymma brevilingue } \\
\text { Schöidte }\end{array}$ & $\begin{array}{l}\text { Sous les rochers et dans les débris végétaux sur la zone littorale ou dans } \\
\text { d'autres milieux humides (Böcher 1988) }\end{array}$ \\
\hline & 2 & Hydroporus morio Aubé & Petits étangs et mares, marais (Böcher 1988) \\
\hline & 3 & $\begin{array}{l}\text { Colymbetes dolabratus } \\
\text { (Payk.) }\end{array}$ & $\begin{array}{l}\text { Étendues d'eau stagnante, surtout celles avec végétation luxuriante } \\
\text { (Böcher 1988) }\end{array}$ \\
\hline & 4 & Bembidion grapii Gyllhenhal & $\begin{array}{l}\text { Sous les pierres et sur des sols relativement secs, souvent près des cours } \\
\text { d'eau avec végétation luxuriante (Böcher 1988) }\end{array}$ \\
\hline & 5 & $\begin{array}{l}\text { Coccinella transversoguttata } \\
\text { Faldermann }\end{array}$ & $\begin{array}{l}\text { Presque tous les types de végétation, plus abondants dans les bruyères } \\
\text { sèches (Böcher 1988) }\end{array}$ \\
\hline
\end{tabular}

certains modes d'exploitation des ressources (traitement des peaux, chasse aux oiseaux, collecte de ressources provenant de nids) qui sont d'un accès difficile au sein des corpus zooarchéologiques et technologiques. L'exploitation et l'importance de diverses ressources végétales sont également décelables dans les assemblages de coléoptères subfossiles. Ces derniers témoignent de l'impact de ces activités sur l'environnement local, causant notamment le déplacement de certaines espèces indigènes et leur prolifération sur les sites d'occupation. De fait, même si les espèces synanthropiques et exotiques communes sur les sites urbains et ruraux sont virtuellement absentes sur les sites paléo- et néoesquimaux, il semble que les conditions environnementales dans leurs habitats n'étaient pas extrêmement différentes de celles des maisons de tourbe norroises de l'Atlantique Nord (Forbes et al. 2014). Dans tous les cas, les assemblages archéoentomologiques des régions arctiques et subarctiques sont indicatifs de conditions humides et d'une abondance de matière organique dans les bâtiments et leurs alentours.

Si les modes de vie des paléo- et des néoesquimaux peuvent laisser des traces discernables dans les archives archéoenvironnementales, l'ampleur et la nature de leur impact sur l'environnement restent à déterminer. L'effet d'enrichissement nutritif apparent sur certaines sites se limite-t-il à l'intérieur des maisons et aux dépotoirs qui leur sont associés, ou affecte-t-il aussi la faune et la flore locales ou régionales? Dans quelle mesure les activités d'exploitation des ressources végétales influencent-elles le paysage? Ce sont là des questions importantes qui font allusion à la capacité probable, mais souvent sous-estimée, des sociétés non agraires à manipuler leurs environnements et à créer des paysages culturels.

\section{Note}

1. Bien que les termes «Thuléens » et «Inuits » soient généralement préférés au Canada pour désigner les ancêtres des Inuits modernes, nous utilisons ici le terme « Néoesquimaux» pour désigner l'ensemble des traditions archéologiques ancestrales non seulement des Inuits du Canada et du Groenland, mais aussi des Yupiks et des Iñupiats de l'Alaska.

\section{Remerciements}

Les auteurs remercient leurs collaborateurs pour la possibilité d'avoir pu recueillir des échantillons archéoentomologiques sur divers sites archéologiques au Canada (William Fitzhugh, Susan Kaplan, Daniel Odess, Douglas Stenton, Marianne Stopp, Patricia Sutherland, Lisa Rankin et James Woollett), au Groenland (Geneviève LeMoine et Christyann Darwent) et en Alaska (Rick Knecht, Kate Britton, Charlotta Hillerdal et Qanirtuuq inc.), ainsi que les communautés inuites et yup'iks qui les ont généreusement 
accueillis. Véronique Forbes aimerait remercier le Arts and Humanities Research Council du Royaume-Uni (University of Aberdeen; AH/K006029/1) pour avoir financé l'écriture de ce manuscrit. Nous remercions aussi deux lecteurs externes pour leurs suggestions et commentaires sur notre manuscrit, ainsi que Frédéric Laugrand pour son invitation à participer à ce numéro thématique.

\section{Ouvrages cités}

AGER, Thomas A., et Lynn PRICE AGER, 1980 : « Ethnobotany of the Eskimos of Nelson Island, Alaska». Arctic Anthropology 17(1): 26-48.

ALIX, Claire, 2004: «Bois flottés et archéologie de l'Arctique: contribution à la préhistoire récente du détroit de Béring ». Études/Inuit/Studies 28(1) : 109-132.

ALIX, Claire, et Karen BREWSTER, 2004 : « Not all driftwood is created equal: Wood use and value along the Yukon and Kuskokwim Rivers, Alaska ». Alaska Journal of Anthropology 2(1) : 48-65.

ANDERSON, J.P., 1939: "Plants used by the Eskimo of the Northern Bering Sea and Arctic regions of Alaska ». American Journal of Botany 26(9) : 714-716.

ARNETT, Ross H. Jr., et Michael C. THOMAS, 2001 : American Beetles, Vol. 1 : Archostemata, Myxophaga, Adephaga, Polyphaga: Staphyliniformia. CRC Press, Boca Raton.

ATKINSON, T.C., et al., 1986: "Climatic calibration of coleopteran data », in Björn E. Berglund (dir.), Handbook of Holocene Palaeoecology and Palaeohydrology: 851-858. J. Wiley \& Sons, Chichester

BAIN, Allison, 1994: Archaeoentomology in the Arctic: coleopteran remains from the Frobisher Site and Willows Island. Rapport non publié, CELAT, Université Laval, Québec.

—, 1997: Coleopteran remains from Mallikjuak Island (LbFn-7) and Peterhead Inlet (KkDo-3), Baffin Island. Rapport non publié, Arctic College, Iqaluit.

—, 1998: «A seventeenth century beetle fauna from colonial Boston ». Historical Archaeology 32 : 38-48

—, 2000 : Uivak archaeoentomological analysis. Rapport non publié, Université Laval, Québec.

—, 2001a: Archaeoentomological and archaeoparasitological reconstructions at Îlot Hunt (CeEt-110): New perspectives in historical archaeology (1850-1900). British Archaeological Reports S973, Archaeopress, Oxford.

—, 2001b: Uivak archaeoentomological analysis-2. Rapport non publié, Université Laval, Québec.

BAIN, Allison L., et Gary KING, 2011 : «Asylum for wayward immigrants: historic ports and colonial settlements in northeast North America ». Journal of the North Atlantic, Special Volume 1 : 109-124.

BAIN, Allison L., et Marie-Annick PRÉVOST, 2010 : «Environmental archaeology and landscape transformations at the $17^{\text {th }}$ century Ferryland site, Newfoundland $»$. Historical Archaeology 44(3) : 21-35

BÖCHER, Jens, 1988 : « The Coleoptera of Greenland ». Meddelelser om Grønland Biosciences 26.

BÖCHER, Jens, et Bent FREDSKILD, 1993: «Plant and arthropod remains from the palaeo-Eskimo site on Qeqertasussuk, West Greenland ». Meddelelser om Grønland Geoscience 30, Copenhagen.

BOUSQUET, Yves, 1990 : Beetles associated with stored products in Canada. An identification guide. Ministry of Supply and Services, Ottawa.

BRESCIANI, J., N. HAARLOV, P. NANSEN et G. MOLLER, 1983 « Head louse (Pediculus humanus subsp. capitis de Geer) from mummified corpses of Greenlanders, A.D. $1460( \pm 50) »$. Acta Entomologica Fennica 42 : 24-27.

—, 1989: "Head Lice in Mummified Greenlanders from A.D. 1475 », in J. P. H. Hansen et H. C. Gulløv (dir.), The Mummies from Qilakitsoq-Eskimos in the 15th Century : 89-92. Meddelelser om Grønland, Man and Society, Copenhagen.

BUCKLAND, Paul C., 1981 : « The early dispersal of insect pests of stored products as indicated by archaeological records ». Journal of Stored Products Research 17 : 1-12.

BUCKLAND, Philip I. et Paul C. BUCKLAND, 2006: BugsCEP Coleopteran Ecology Package. IGBP PAGES/World Data Center for Paleoclimatology Data Contribution Series \# 2006-116. NOAA/ NCDC Paleoclimatology Program, Boulder. <http://www.ncdc. noaa.gov/paleo/insect.html \& http://www.bugscep.com> (consulté le $1^{\text {er }}$ août 2016).

BUCKLAND, P.C., P. BUCKLAND et P. SKIDMORE, 1998 : «Insect remains from GUS: an interim report », in Jette Arneborg et Hans Christian Gulløv (dir.), Man, culture and environment in ancient Greenland: 74-79. Dansk Polar Centre et Danish National Museum, Copenhagen.

BUCKLAND, Paul C., et Jon P. SADLER, 1989 : « A biogeography of the human flea, Pulex irritans L. (Siphonaptera: Pulicidae)». Journal of Biogeography 16(2) : 115-120.

BUCKLAND, P.C., J. SADLER et G. SVEINBJARNARDÓTTIR, 1992 : «Palaeoecological investigations at Reykholt, Western Iceland », in Christopher D. Morris et D.J. Rackham (dir.), Norse and later settlement and subsistence in the North Atlantic: 149-167. University of Glasgow, Glasgow.

CAMPBELL, J.M., 1979: A revision of the genus Tachyporus Gravenhorst (Coleoptera: Staphylinidae) of North and Central America. Memoirs of the Entomological Society of Canada 109, Ottawa.

—, 1982 : «A revision of the North American Omaliinae (Coleoptera: Staphylinidae). 3. The genus Acidota Stephens ». The Canadian Entomologist 114(11) : 1003-1029.

—, 1983: "A new species of Pycnoglypta Thomson (Coleoptera: Staphylinidae) from eastern Canada ». The Canadian Entomologist $115: 361-370$.

—, 1984 : «A revision of the North American Omaliinae (Coleoptera: Staphylinidae). The genera Arpedium Erichson and Eucnecosum Reitter ». The Canadian Entomologist 116 : 487-527.

COOPE, G. Russell, 1978: «Constancy of insect species versus inconstancy of Quaternary environments », in L. A. Mound et N. Waloff (dir.), Diversity of Insect Faunas. Symposia of the Royal Entomological Society of London 9 : 179-187. Blackwell Scientific Publications, Oxford.

COOPE, G. Russell, et Peter J. OSBORNE, 1967 : « Report on the coleopterous fauna of the Roman well at Barnsley Park, Gloucestershire ». Transactions of the Bristol and Gloucestershire Archaeological Society $86:$ 84-87.

COOPE, G. Russell, et al., 1977 : « Fossil Coleoptera assemblages as sensitive indicators of climatic changes during the Devensian (Last) Cold Stage ». Philosophical Transactions of the Royal Society of London 280 : 313-340.

CRAWFORD, Laura J., 2012: Thule plant and fuel use at Cape Espenberge, Alaska. Ph.D. Thesis, University of Alaska Fairbanks.

CROWSON, Roy A., 1981 : The Biology of the Coleoptera. Academic Press, London.

DURDEN, Lance A., 2002 : « Lice (Phthiraptera) », in Gary Mullen et Lance A. Durden (dir.), Medical and Veterinary Entomology: 45-65. Academic Press, San Diego.

DUSSAULT, Frédéric, et Allison BAIN, 2010: Assessment of the entomological remains from the North Island (FeAx-3) and Great 
Caribou Island (FbAv-13) sites, Labrador. Rapport non publié, Université Laval, Québec.

DUSSAUlT, F, A. BAIN et G. LEMOINE, 2014: "Early Thule winter houses: An archaeoentomological analysis». Arctic Anthropology 51(1) : 101-117.

DUSSAULT,F., T.J.BELLetV.GRIMES,2016 : A Archaeoentomological perspectives on Dorset occupations in Newfoundland: A case study from the site of Phillip's Garden (EeBi-1)». Arctic 69(suppl. 1) : 1-15.

ELIAS, Scott A., 2003: "Past climates at Uivvaq based on its insect fauna », in Owen K. Mason (dir.), Uivvaq Heritage Project - Field Season 2002: 67-71. Final Report to Aglaq CON/AM J.V. $\mathrm{N}^{\circ} 2$, Cape Lisburne Clean Sweep Remedial Action, Geoarch Alaska, Anchorage.

—, 2010 : Advances in Quaternary entomology. Elsevier, Amsterdam.

ELIAS, Scott A., et Jon V. MATTHEWS Jr., 2002 : « Arctic North American seasonal temperatures from the latest Miocene to the early Pleistocene, based on mutual climatic range analysis of fossil beetle assemblages. Canadian Journal of Earth Sciences 39: 911-920.

FIENUP-RIORDAN, Ann, 2007: Yuungnaqpiallerput (the way we genuinely live): Masterworks of Yup'ik science and survival. University of Washington Press, Seattle.

FORBES, Véronique, 2015 : «Duck fleas as evidence for eiderdown production on archaeological sites ». Journal of Archaeological Science 61 : 105-111.

FORBES, V., K. BRITTON et R. KNECHT, 2015: "Preliminary archaeoentomological analyses of permafrost-preserved cultural layers from the pre-contact Yup'ik Eskimo site of Nunalleq, Alaska: implications, potential and methodological considerations ». Environmental Archaeology 20(2) : 158-167.

FORBES, V., A.J. DUGMORE et E. ÓLAFSSON, 2016 : «The life and death of barn beetles: Faunas from manure and stored hay inside farm buildings in northern Iceland ». Ecological Entomology 41 : 480-499.

FORBES, Véronique, Frédéric DUSSAULT et Allison BAIN, 2013 : "Contributions of ectoparasite studies in archaeology with two examples from the North Atlantic region ». International Journal of Paleopathology 3 : 158-164.

—, 2014: "Archaeoentomological research in the North Atlantic: Past, present, and future ». Journal of the North Atlantic 26 : 1-24.

FORBES, Véronique, et Karen B. MILEK, 2014 : «Insects, activity areas and turf buildings' interiors: An ethno-archaeoentomological case study from $19^{\text {th }}$ to early $20^{\text {th }}$-century Pverá, northeast-Iceland ». Quaternary International 341 : 195-215.

FRIESEN, T. Max, 2004 : « Contemporaneity of Dorset and Thule cultures in the North American Arctic: new radiocarbon dates from Victoria Island, Nunavut ». Current Anthropology 45(5): 685-691.

FRIESEN, T. Max, et Owen K. MASON, (dir.), 2016: The Oxford Handbook of the Prehistoric Arctic. Oxford University Press, Oxford.

GRØNNOW, Bjarne, (dir.), 2009: On the track of the Thule culture from Bering Strait to east Greenland. Proceedings of the SILA conference "The Thule culture - new perspectives on Inuit prehistory", Copenhagen, Oct. 26th-28th, 2006. Papers in honour of Hans Christian Gulløv. National Museum of Denmark, Copenhagen.

HAARL $\varnothing V$, N., 1967: "Arthropoda (Acarina, Diptera) from subfossil layers in west Greenland ». Meddelelser om Grønland 184(3) : 1-17.

HANSEN, J.P.H., J. MELDGAARD et J. NORDQVIST, 1991: The Greenland Mummies. British Museum Publications, London.

HATCH, Melville Harrison, 1957 : The beetles of the Pacific Northwest. Part II: Staphyliniformia. University of Washington Publications in Biology 16. University of Washington Press, Seattle.
HELBAEK, H., 1970: «The plant husbandry of Haçilar», in J. Melaart (dir.), Excavations at Haçilar 1: 189-244. Edinburgh University Press, Edinburgh.

HENSON, Matthew A., Robert E. PEARY et A. FREDERICK, 1912 : A negro explorer at the north pole. Stokes, New York.

HINTON, H.E., 1941: "The Lathridiidae of economic importance ». Bulletin of Entomological Research 32(3) : 191-247.

HOFFECKER, John F., 2005 : A prehistory of the north: Human settlements of the higher latitudes. Rutgers University Press, New Brunswick.

HOFFECKER, John F., et al., 2012 : « Uivvaq: A stratified Iñupiaq occupation at Cape Lisburne, northwest Alaska». Alaska Journal of Anthropology 10(1-2) : 143-172.

HOLLAND, George P., 1985: The fleas of Canada, Alaska and Greenland (Siphonaptera). Memoirs of the Entomological Society of Canada 130, Ottawa.

HOPLA, C.E., L.A. DURDEN et J.E. KEIRANS, 1994 : « Ectoparasites and classification ». Revue scientifique et technique Office international des Épizooties 13 : 985-1017.

HUCHET, Jean-Bernard, 1996: «Larchéoentomologie funéraire: une approche originale dans l'interprétation des sépultures». Bulletins et Mémoires de la Société d'anthropologie de Paris, Nouvelle Série 8(3-4) : 299-311.

INSTITUT CULTUREL AVATAQ, 2015 : Chronologie de l'Arctique. <http://www.avataq.qc.ca/fr/L-institut/Departements/ Archeologie/Decouvrir-l-archeologie/Chronologie-de-lArctique> (consulté le 8 novembre 2016).

KAPLAN, Susan A., et James M. WOOLLETT, 2000 : «Challenges and choices: Exploring the interplay of climate, history and culture on Canada's Labrador coast ». Arctic, Antarctic and Alpine Research 32(3): 351-359.

KENWARD, Harry, 2009 : Northern regional review of environmental archaeology: Invertebrates in archaeology in the north of England. Research Department Report Series (12/2009), English Heritage, Portsmouth.

KENWARD, Harry, et Enid ALLISON, 1994 : « Rural origins of the urban insect fauna », in Allan R. Hall et Harry K. Kenward (dir.), Urban-rural connexions: Perspectives from environmental archaeology : 55-78. Oxbow Monograph 47, Oxford

KING, Gary A., 2014 : «Exaptation and synanthropic insects: A diachronic interplay between biology and culture ». Environmental Archaeology 19(1) : 12-22.

KLEIVAN, H., 1978 : « Greenland Eskimo: Introduction », in David Damas (ed.) Handbook of North American Indians : Arctic (vol. 5). Smithsonian Institution Press, Washington.

KLIMASZEWSKI, Jan, et al., 2010: Review of adventive species of Coleoptera (Insecta) recorded from eastern Canada. Pensoft Publishers, Bulgaria.

LALONDE, Olivier, 2015 : Assessment of insect remains from a communal house at the Double Mer Point site (GbBo-2), Labrador. Rapport non publié, GAIA et Memorial University of Newfoundland.

—, 2016: Analysis of entomological remains from two communal houses at the Double Mer Point site (GbBo-2), Labrador. Rapport non publié, GAIA et Memorial University of Newfoundland.

LAUGRAND, Frédéric, et Jarich OOSTEN, 2010 : « Qupirruit: Insects and worms in Inuit traditions ». Arctic Anthropology 47(1) : 1-21.

LAVOIE, Claude, et Dominique ARSENEAULT, 2001: «Late Holocene climate of the James Bay area, Québec, Canada, reconstructed using fossil beetles ». Arctic, Antarctic, and Alpine Research $33:$ 13-18. 
LEMUS-LAUZON, I., N. BHIRY, et J. WOOLLETT, 2012: « Napâttuit: Wood use by Labrador Inuit and its impact on the forest landscape ». Études/Inuit/Studies 36(1) : 113-137.

_, 2016: «Assessing the effects of climate change and land use on northern Labrador forest stands based on paleoecological data». Quaternary Research. <http://dx.doi.org/10.1016/j. yqres.2016.09.001>

LEPOFSKY, D., M.L. MOSS, et N. LYONS, 2001 : « The unrealized potential of paleoethnobotany in the archaeology of northwestern North America: Perspectives from Cape Addington, Alaska ». Arctic Anthropology 38(1) : 48-59.

MAJKA, C.G., D. LANGOR, et W.H. RÜCKER, 2009 : « Latridiidae (Coleoptera) of Atlantic Canada: New records, keys to identification, new synonyms, distribution, and zoogeography». Canadian Entomologist 141 : 317-370.

MARSHALL, Adrian G., 1981: The ecology of ectoparasitic insects. Academic Press, London.

MASCHNER, Herbert, Owen MASON et Robert McGHEE, (dir.), 2009: The northern world AD 900-1400. The University of Utah Press, Salt Lake City.

McGHEE, Robert, 2001 : Ancient peoples of the Arctic. UBC Press, Vancouver.

McMANUS, Ellen T., 2015 : Pre-contact ecology, subsistence and diet on the Yukon-Kuskokwim Delta. An integrated ecosystem approach to pre-contact Arctic lifeways using zooarchaeological analysis and stable isotope techniques. Ph.D. Thesis, University of Aberdeen.

NIELSEN, B.O., V. MAHLER et P. RASMUSSEN, 2000 : «An arthropod assemblage and the ecological conditions in a byre at the Neolithic settlement of Weier, Switzerland». Journal of Archaeological Science 27 : 209-218.

OBATA, H., et al., 2011 : «A new light on the evolution and propagation of prehistoric grain pests: The world's oldest maize weevils found in Jomon potteries, Japan ». PLoS ONE 6(3) : e14785.

PARK, Robert W., 1993: "The Dorset-Thule succession in arctic North America: Assessing claims for culture contact ». American Antiquity 58(2) : 203-234.

—, 1999 : «Demography and the reconstruction of social organization from Thule wintering sites in Arctic Canada». Canadian Journal of Archaeology 22(2) : 115-126.

PEARY, Robert E., 1898 : Northward over the Great Ice: A narrative of life and work along the shores and upon the interior ice-cap of northern Greenland in the years 1886 and 1891-1897. F.A. Stokes Company, New York (2 volumes).

PEARY, Josephine D., et Robert E. PEARY, 1893 : My Arctic Journal: A year among the ice-fields and Eskimos. Contemporary Publishing Company, New York.

PINTAL, Jean-Yves, Gisèle PIÉDALUE et Jean PROVENCHER, 2015: AIR - Archéologie du Québec: territoire et peuplement. Pointe-à-Callière et Éditions de l'Homme, Montréal.

PLARRE, Rudy, 2010 : «An attempt to reconstruct the natural and cultural history of the granary weevil, Sitophilus granarius (Coleoptera: Curculionidae) ». European Journal of Entomology 107(1-11) : 1-11.

PONEL, Philippe, et al., 2013 : « Fossil beetles as possible evidence for transhumance during the middle and late Holocene in the high mountains of Talysch (Talesh) in NW Iran? » Environmental Archaeology 18(3) : 201-210.

PONEL, Philippe, et al., 2005: «Lateglacial palaeoenvironments and palaeoclimates from Conty and Houdancourt, northern France, reconstructed from beetle remains ». Quaternary Science Reviews 24 : 2449-2465.
RAGHAVAN, Maanasa, et al., 2014 : « The genetic prehistory of the New World Arctic ». Science 345(6200).

RASMUSSEN, Knud, 1929: Du Groenland au Pacifique: deux ans d'intimité avec des tribus d'esquimaux inconnus. Traduit par C. Lund et J. Bernard. Plon, Paris.

RENOUF, M.A. Patricia, 1999: «Prehistory of Newfoundland hunter-gatherers: Extinctions or adaptations? » World Archaeology 30(3) : 403-420.

—, 2003: «A review of Palaeoeskimo dwelling structures in Newfoundland and Labrador ». Études/Inuit/Studies 27(1-2): 375-416.

—, 2011 : The cultural landscapes of Port au Choix precontact huntergatherers of northwestern Newfoundland. Springer, New York.

RENOUF, M.A. Patricia, et Trevor BELL, 2009: «Contraction and expansion in Newfoundland prehistory, AD 900-1500 », in H. Maschner, O. Mason et R. McGhee (dir.), The northern world AD 900-1400 : 263-278. The University of Utah Press, Salt Lake City.

ROTHSCHILD, Miriam, et Teresa CLAY, 1957 : Fleas, flukes and cuckoos. A Study of Bird Parasites. The MacMillan Company, New York.

ROY, Natasha, Najat BHIRY et James WOOLLETT, 2012: " Environmental change and terrestrial resource use by the Thule and Inuit of Labrador, Canada ». Geoarchaeology 27 : 18-33.

ROY, Natasha, James WOOLLETT et Najat BHIRY, 2015: «Paleoecological perspectives on landscape history and anthropogenic impacts at Uivak Point, Labrador, since AD $1400 »$. The Holocene 25(11) : 1742-1755.

SCHELDERMANN, Peter, 1976 : « Thule culture communal houses in Labrador ». Arctic 29(1): 27-37.

SMETANA, Aleš, 1988 : Review of the family Hydrophilidae of Canada and Alaska (Coleoptera). Memoirs of the Entomological Society of Canada 142, Ottawa.

SMITH, David N., 1992 : «The Coleopterous faunas from Kuyait and Kamaiyuk, Baffin Island, Canada », in Archaeology of the Frobisher voyages: regional surveys and European-Inuit contact studies. Smithsonian Institution, Washington D.C.

—, 1994 : The insect remains from the 1992 excavations at Kamaiyuk and Kuyait, Baffin Island, Canada. Rapport non publié, Harvard University, Cambridge.

—, 2012: Insects in the city: An archaeoentomological perspective on London's past. British Archaeological Reports, British Series 561. Archaeopress, Oxford.

TAYLOR, J. Garth, 1974: Labrador Eskimo settlements of the early contact period. National Museums of Canada, Ottawa.

WOOLLETT, James, 2007: «Labrador Inuit subsistence in the context of environmental change: An initial landscape history perspective ». American Anthropologist 109(5) : 69-84.

YVINEC, Jean Hervé, Philippe PONEL, et Jean-Claude BEAUCOURNU, 2000: "Premier apports archéoentomologiques de l'étude des puces : aspects historiques et anthropologiques (Siphonaptera) ». Bulletin de la Société entomologique de France 105(4) : 419-425

ZUTTER, Cynthia, 2009: «Paleoethnobotanical contributions to $18^{\text {th }}$ century Inuit economy: An example from Uivak, Labrador ». Journal of the North Atlantic 1 : 23-32.

—, 2012: «The shrubs in the forest: The use of woody species by 18th-century Labrador Inuit ». Études/Inuit/Studies 36(1): 139-155. 